\title{
A unification of permutation patterns related to Schubert varieties
}

\author{
Henning A. Úlfarsson $\|^{\dagger}$ \\ Mathematics Institute, School of Computer Science, Reykjavik University, Reykjavik, Iceland
}

\begin{abstract}
We prove new connections between permutation patterns and singularities of Schubert varieties, by giving a new characterization of factorial and Gorenstein varieties in terms of so called bivincular patterns. These are generalizations of classical patterns where conditions are placed on the location of an occurrence in a permutation, as well as on the values in the occurrence. This clarifies what happens when the requirement of smoothness is weakened to factoriality and further to Gorensteinness, extending work of Bousquet-Mélou and Butler (2007), and Woo and Yong (2006). We also prove results that translate some known patterns in the literature into bivincular patterns.

Résumé. Nous démontrons de nouveaux liens entre les motifs de permutation et les singularités des variétés de Schubert, par la méthode de donner une nouvelle caractérisation des variétés factorielles et de Gorenstein par rapport à les motifs bivinculaires. Ces motifs sont généralisations des motifs classiques où des conditions se posent sur la position d'une occurrence dans une permutation, aussi bien que sur les valeurs qui se présentent dans l'occurrence. Ceci éclaircit les phénomènes où la condition de nonsingularité s'affaiblit á factorialité et même à Gorensteinité, et augmente les travaux de Bousquet-Mélou et Butler (2007), et de Woo et Yong (2006). Nous démontrons également des résultats qui traduisent quelques motifs connus en la littérature en motifs bivinculaires.
\end{abstract}

Keywords: Patterns, Permutations, Schubert varieties, Singularities

\section{Introduction}

We prove new connections between permutation patterns and singularities of Schubert varieties $X_{\pi}$ in the complete flag variety Flags $\left(\mathbb{C}^{n}\right)$, by giving a new characterization of factorial and Gorenstein varieties in terms of which bivincular patterns the permutation $\pi$ avoids. Bivincular patterns, defined by Bousquet-Mélou et al. (2010), are generalizations of classical patterns where conditions are placed on the location of an occurrence in a permutation, as well as on the values in the occurrence. This clarifies what happens when the requirement of smoothness is weakened to factoriality and further to Gorensteinness, extending work of Bousquet-Mélou and Butler (2007), and Woo and Yong (2006). We also prove results that translate some known patterns in the literature into bivincular patterns. In particular we will give a characterization of the Baxter permutations.

In section 2 we recall the definitions of classical, vincular (also called generalized patterns, BabsonSteingrímsson patterns or dashed patterns), bivincular and barred patterns. We will also recall Bruhat embeddings of patterns, as defined by Woo and Yong (2006).

\footnotetext{
${ }^{\dagger}$ This work is supported by grant no. 090038011 from the Icelandic Research Fund. 
In section 3 we will show how these different types of patterns and their avoidances are related to each other. We will then use patterns that come up in the connections between permutations and Schubert varieties as motivation. In particular, recall the theorem of Ryan (1987), Wolper (1989) and Lakshmibai and Sandhya (1990) that the Schubert variety $X_{\pi}$ is non-singular (or smooth) if and only if $\pi$ avoids the patterns 1324 and 2143 . Saying that the variety $X_{\pi}$ is non-singular means that every local ring is regular. A weakening of this condition is the requirement that every local ring only be a unique factorization domain; a variety satisfying this is a factorial variety.

Bousquet-Mélou and Butler (2007) proved a conjecture stated by Yong and Woo (Bousquet-Mélou et al. 2005, Personal communication) that factorial Schubert varieties are those that correspond to permutations avoiding 1324 and bar-avoiding 21354. In the terminology of Woo and Yong (2006) the baravoidance of the latter pattern corresponds to avoiding 2143 with Bruhat condition $(1 \leftrightarrow 4)$, or equivalently, interval avoiding [2413, 2143] in the terminology of Woo and Yong (2008). However, as remarked by Steingrímsson (2007), bar-avoiding $21 \overline{3} 54$ is equivalent to avoiding the vincular pattern $2 \underline{14} 3$. If we summarize this in terms of vincular patterns a striking thing becomes apparent; see the lines corresponding to smoothness and factoriality in Table 1 , and Theorem 3.1 .

\begin{tabular}{|c|c|}
\hline$X_{\pi}$ is & The permutation $\pi$ avoids the patterns \\
\hline smooth & 2143 and 1324 \\
\hline factorial & $2 \underline{14} 3$ and 1324 \\
\hline Gorenstein & $\begin{array}{l}1 \overline{2 \overline{34}} 5,1 \overline{23} 45 \\
31524,24153 \text {; and satisfies a condition on } \\
\text { descents involving two infinite families of bivincular patterns }\end{array}$ \\
\hline
\end{tabular}

Tab. 1: Connections between singularity properties and bivincular patterns. See Theorem 3.1 for the second line and see Theorem 3.13 for the third line.

We see that requiring 1 and 4 to be adjacent in the first pattern (and thus turning it into a vincular pattern) corresponds to weakening smoothness to factoriality.

A further weakening is to only require that the local rings of $X_{\pi}$ be Gorenstein local rings, in which case we say that $X_{\pi}$ is a Gorenstein variety. Woo and Yong (2006) showed that $X_{\pi}$ is Gorenstein if and only if it avoids two patterns with two Bruhat restrictions each, as well as satisfying a certain condition on descents. We will translate their results into avoidance of bivincular patterns; see Theorem 3.13 in subsection 3.2 and the line corresponding to Gorensteinness in Table 11. Essentially the two bivincular patterns that are shown there are certain upgrades of the pattern $2 \underline{14} 3$ while the avoidance of the pattern 1324 is weakened to avoidance of two infinite families of bivincular patterns.

In section 3 we will also prove a proposition that leads to a characterization of the Baxter permutations in terms of vincular patterns, see Example 3.5

\section{Three Types of Pattern Avoidance}

Here we recall definitions of different types of patterns. We will use one-line notation for all permutations, e.g., write $\pi=312$ for the permutation in $S_{3}$ that satisfies $\pi(1)=3, \pi(2)=1$ and $\pi(3)=2$.

The three types correspond to:

- Bivincular patterns, subsuming vincular patterns and classical patterns. 
- Barred patterns.

- Bruhat restricted patterns.

\subsection{Bivincular Patterns}

We denote the symmetric group on $n$ letters by $S_{n}$, whose elements are permutations. We write permutations as words $\pi=a_{1} a_{2} \cdots a_{n}$, where the letters are distinct and come from the set $\{1,2, \ldots, n\}$. A pattern $p$ is also a permutation, but we are interested in when a pattern is contained in a permutation $\pi$ as described below.

An occurrence (or embedding) of a pattern $p$ in a permutation $\pi$ is classically defined as a subsequence in $\pi$, of the same length as $p$, whose letters are in the same relative order (with respect to size) as those in $p$. For example, the pattern 123 corresponds to a increasing subsequence of three letters in a permutation. If we use the notation $1_{\pi}$ to denote the first, $2_{\pi}$ for the second and $3_{\pi}$ for the third letter in an occurrence, then we are simply requiring that $1_{\pi}<2_{\pi}<3_{\pi}$. If a permutation has no occurrence of a pattern $p$ we say that $\pi$ avoids $p$.

Example 2.1 The permutation 32415 contains two occurrences of the pattern 123 corresponding to the sub-words 345 and 245. It avoids the pattern 132.

In a vincular pattern (also called a generalized pattern, Babson-Steingrímsson pattern or dashed pattern), two adjacent letters may or may not be underlined. If they are underlined it means that the corresponding letters in the permutation $\pi$ must be adjacent.

Example 2.2 The permutation 32415 contains one occurrence of the pattern 123 corresponding to the sub-word 245. It avoids the pattern $1 \underline{23}$. The permutation $\pi=324615$ has one occurrence of the pattern 2143 , namely the sub-word 3265, but no occurrence of 2143 , since 2 and 6 are not adjacent in $\pi$.

These types of patterns have been studied sporadically for a very long time but were not defined in full generality until Babson and Steingrímsson (2000).

This notion was generalized further in Bousquet-Mélou et al. (2010): In a bivincular pattern we are also allowed to put restrictions on the values that occur in an embedding of a pattern. We use two-line notation to describe these patterns. If there is a line over the letters $i, i+1$ in the top row, it means that the corresponding letters in an occurrence must be adjacent in values. This is best described by an example:

Example 2.3 An occurrence of the pattern 123 in a permutation $\pi$ is an increasing subsequence of three letters, such that the second one is larger than the first by exactly 1 , or more simply $2_{\pi}=1_{\pi}+1$. The permutation 32415 contains one occurrence of this bivincular pattern corresponding to the sub-word 345 .

This is also an occurrence of $\overline{123}$. The permutation avoids the bivincular pattern $\overline{123}$.

We will also use the notation of Bousquet-Mélou et al. (2010) to write bivincular patterns: A bivincular pattern consists of a triple $(p, X, Y)$ where $p$ is a permutation in $S_{k}$ and $X, Y$ are subsets of $\llbracket 0, k \rrbracket$. An occurrence of this bivincular pattern in a permutation $\pi=\pi_{1} \cdots \pi_{n}$ in $S_{n}$ is a subsequence $\pi_{i_{1}} \cdots \pi_{i_{k}}$ such that the letters in the subsequence are in the same relative order as the letters of $p$ and

- for all $x$ in $X, i_{x+1}=i_{x}+1$; and

- for all $y$ in $Y, j_{y+1}=j_{y}+1$, where $\left\{\pi_{i_{1}}, \ldots, \pi_{i_{k}}\right\}=\left\{j_{1}, \ldots, j_{k}\right\}$ and $j_{1}<j_{2}<\cdots<j_{k}$. 
By convention we put $i_{0}=0=j_{0}$ and $i_{k+1}=n+1=j_{k+1}$.

Example 2.4 We can translate all of the patterns we have discussed above into this notation:

$$
\begin{aligned}
& 123=(123, \varnothing, \varnothing), \quad 132=(132, \varnothing, \varnothing), \quad \underline{12} 3=(123,\{1\}, \varnothing), \\
& 1 \underline{23}=(123,\{2\}, \varnothing), \quad 2143=(2143, \varnothing, \varnothing), \quad 2 \underline{14} 3=(2143,\{2\}, \varnothing), \\
& \begin{array}{ll}
\overline{12} 3 \\
123=(123, \varnothing,\{1\}), & \overline{123}=(123, \varnothing,\{1,2\}), \quad \overline{123}
\end{array}=(123,\{2\},\{1,2\}) .
\end{aligned}
$$

We have not considered the case when 0 or $k$ are elements of $X$ or $Y$, as we will not need those cases. We just remark that if $0 \in X$ then an occurrence of $(p, X, Y)$ must start at the beginning of a permutation $\pi$, in other words, $\pi_{i_{1}}=\pi_{1}$. The other cases are similar.

The bivincular patterns behave well with respect to the operations reverse, complement and inverse: Given a bivincular pattern $(p, X, Y)$ we define

$$
(p, X, Y)^{\mathrm{r}}=\left(p^{\mathrm{r}}, k-X, Y\right), \quad(p, X, Y)^{\mathrm{c}}=\left(p^{\mathrm{c}}, X, k-Y\right), \quad(p, X, Y)^{\mathrm{i}}=\left(p^{\mathrm{i}}, Y, X\right),
$$

where $p^{\mathrm{r}}$ is the usual reverse of the permutation of $p, p^{\mathrm{c}}$ is the usual complement of the permutation of $p$, and $p^{\mathrm{i}}$ is the usual inverse of the permutation of $p$. Here $k-M=\{k-m \mid m \in M\}$.

We get a very simple but useful lemma:

Lemma 2.5 Let a denote one of the operations above (or their compositions). Then a permutation $\pi$ avoids the bivincular pattern $p$ if and only if the permutation $\pi^{\mathrm{a}}$ avoids the bivincular pattern $p^{\mathrm{a}}$.

\subsection{Barred Patterns}

We will only consider a single pattern of this type, but the general definition is easily inferred from this special case. We say that a permutation $\pi$ avoids the barred pattern $21 \overline{3} 54$ if $\pi$ avoids the pattern 2143 (corresponding to the unbarred elements) except where that pattern is a part of the pattern 21354. This notation for barred patterns was introduced by West (1990). It turns out that avoiding this barred pattern is equivalent to avoiding $2 \underline{14} 3$, see section 3 .

Example 2.6 The permutation $\pi=425761$ avoids the barred pattern $21 \overline{3} 54$ since the unique occurrence of 2143, as the sub-word 4276, is contained in the sub-word 42576 which is an occurrence of 21354.

\subsection{Bruhat Restricted Patterns}

Here we recall the definition of Bruhat restricted patterns from Woo and Yong (2006). First we need the Bruhat order on permutations in $S_{n}$, defined as follows: Given integers $i<j$ in $\llbracket 1, n \rrbracket$ and a permutation $\pi \in S_{n}$ we define $w(i \leftrightarrow j)$ as the permutation that we get from $\pi$ by swapping $\pi(i)$ and $\pi(j)$. For example $24153(1 \leftrightarrow 4)=54123$. We then say that $\pi(i \leftrightarrow j)$ covers $\pi$ if $\pi(i)<\pi(j)$ and for every $k$ with $i<k<j$ we have either $\pi(k)<\pi(i)$ or $\pi(k)>\pi(j)$. We then define the Bruhat order as the transitive closure of the above covering relation. This definition should be compared to the construction of the graph $G_{\pi}$ in subsection 3.1. We see that in our example above that 24153(1 $\left.\leftrightarrow 4\right)$ does not cover 24153 since we have $\pi(2)=4$. Now, given a pattern $p$ with a set of transpositions $\mathcal{T}=\left\{\left(i_{\ell} \leftrightarrow j_{\ell}\right)\right\}$ we say that a permutation $\pi$ contains $(p, \mathcal{T})$, or that $\pi$ contains the Bruhat restricted pattern $p$, if $\mathcal{T}$ is understood from the context, if there is an embedding of $p$ in $\pi$ such that if any of the transpositions in $\mathcal{T}$ are carried out on the embedding the resulting permutation covers $\pi$. 
We should note that Bruhat restricted patterns were further generalized to intervals of patterns in Woo and Yong (2008). We will not consider this generalization here.

In the next section we will show how these three types of patterns are related to one another.

\section{Connections between the Three Types}

\subsection{Factorial Schubert Varieties and Forest-like Permutations}

Bousquet-Mélou and Butler (2007) defined and studied forest-like permutations. Here we recall their definition: Given a permutation $\pi$ in $S_{n}$, construct a graph $G_{\pi}$ on the vertex set $\{1,2, \ldots, n\}$ by joining $i$ and $j$ if

1. $i<j$ and $\pi(i)<\pi(j)$; and

2. there is no $k$ such that $i<k<j$ and $\pi(i)<\pi(k)<\pi(j)$.

The permutation $\pi$ is forest-like if the graph $G_{\pi}$ is a forest. In light of the definition of Bruhat covering above we see that the vertices $i$ and $j$ are connected in the graph of $G_{\pi}$ if and only if $\pi(i \leftrightarrow j)$ covers $\pi$.

They then show that a permutation is forest-like if and only if it avoids the classical pattern 1324 and the barred pattern $p_{\text {bar }}=21 \overline{3} 54$. This barred pattern can be described in terms of Bruhat restricted embeddings and in terms of bivincular patterns, as we now show.

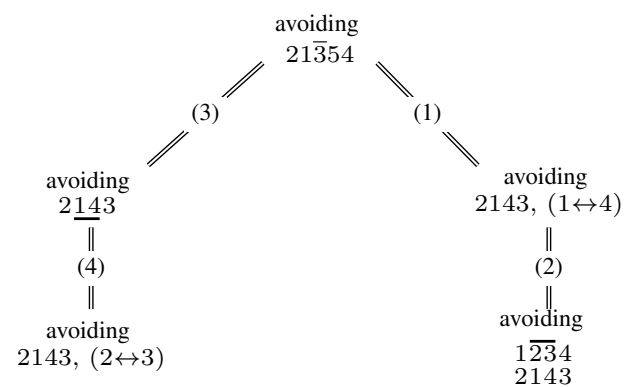

Fig. 1: The barred pattern $21 \overline{3} 54$ gives a connection between two bivincular patterns. The labels on the edges correspond to the enumerated list below.

1. Bousquet-Mélou and Butler (2007) remark that forest-like permutations $\pi$ correspond to factorial Schubert varieties $X_{\pi}$ and avoiding the barred pattern is equivalent to avoiding $p_{\mathrm{Br}}=2143$ with Bruhat restriction $(1 \leftrightarrow 4)$. This last part is easily verified.

2. Avoiding $p_{\mathrm{Br}}=2143$ with Bruhat restriction $(1 \leftrightarrow 4)$ is equivalent to avoiding the bivincular pattern $p_{\text {bi }}=\begin{aligned} & 1 \overline{23} 4 \\ & 2143\end{aligned}$, as we will now show:

Assume $\pi$ contains the bivincular pattern $p_{\text {bi }}$, so we can find an embedding of it in $\pi$ such that $3_{\pi}=2_{\pi}+1$. This embedding clearly satisfies the Bruhat restriction. 
Now assume that $\pi$ has an embedding of $p_{\mathrm{Br}}$. If $3_{\pi}=2_{\pi}+1$ we are done. Otherwise $2_{\pi}+1$ is either to the right of $3_{\pi}$ or to the left of $2_{\pi}$ (because of the Bruhat restriction). In the first case change $3_{\pi}$ to $2_{\pi}+1$ and we are done. In the second case replace $2_{\pi}$ with $2_{\pi}+1$, thus reducing the distance in values to $3_{\pi}$, then repeat.

This will be generalized in Proposition 3.8 .

3. The barred pattern $p_{\text {bar }}=21 \overline{3} 54$ has another connection to bivincular patterns: avoiding it is equivalent to avoiding the bivincular pattern $q_{\text {biv }}=2 \underline{14} 3$, as remarked in the survey by Steingrímsson (2007).

4. We can translate this into Bruhat restricted embeddings as well: Avoiding the bivincular pattern $q_{\mathrm{bi}}=2 \underline{14} 3$ is equivalent to avoiding $q_{\mathrm{Br}}=2143$ with Bruhat restriction $(2 \leftrightarrow 3)$ :

Assume $\pi$ has an embedding of $q_{\mathrm{Br}}$. If $1_{\pi}$ and $4_{\pi}$ are adjacent then we are done. Otherwise look at the letter to right of $1_{\pi}$. If this letter is larger than $4_{w}$ we can replace $4_{w}$ by it and we are done. Otherwise this letter must be less than $4_{w}$, which implies by the Bruhat restriction, that it must also be less than $1_{w}$. In this case we replace $1_{w}$ by this letter, and repeat.

Now assume $\pi$ has an embedding of the bivincular pattern $q_{\mathrm{bi}}$. If $1_{\pi}$ and $4_{\pi}$ are adjacent we are done. Otherwise look at the letter to the right of $1_{\pi}$. This letter is either smaller than $1_{\pi}$ or larger than $4_{\pi}$. In the first case, replace $1_{\pi}$ with this letter; in the second case, replace $4_{\pi}$ with this letter. Then repeat if necessary.

This will be generalized in Proposition 3.8 .

This gives us:

Theorem 3.1 Let $\pi \in S_{n}$. The Schubert variety $X_{\pi}$ is factorial if and only if $\pi$ avoids the patterns $2 \underline{14} 3$ and 1324 .

From the equivalence of the patterns in Figure 1 we also get that a permutation $\pi$ avoids the bivincular pattern

$$
2 \underline{14} 3=(2143,\{2\}, \varnothing)
$$

if and only if it avoids

$$
{ }_{2143}^{123}=(2143, \varnothing,\{2\}) .
$$

We will prove this without going through the barred pattern, and then generalize the proof, but first of all we should note that these bivincular patterns are inverses of one another, and that will simplify the proof.

Assume $\pi$ contains $1 \overline{23} 4$. If $1_{\pi}$ and $4_{\pi}$ are adjacent in $\pi$ we are done. Otherwise consider the element immediately to the right of $1_{\pi}$. If this element is less than $2_{\pi}$ then replace $1_{\pi}$ by it and we will have reduced the distance between $1_{\pi}$ and $4_{\pi}$. If this element is larger than $2_{\pi}$ it must also be larger than $3_{\pi}$, since $3_{\pi}=2_{\pi}+1$, so replace $4_{\pi}$ by it. This will (immediately, or after several steps) produce an occurrence of $2 \underline{14} 3$.

Now assume $\pi$ contains $2 \underline{14} 3$. Then $\pi^{\mathrm{i}}$ contains the inverse pattern

$$
(2 \underline{14} 3)^{\mathrm{i}}=\begin{aligned}
& 1 \overline{23} 4 \\
& 2143
\end{aligned} .
$$


Then by the above, $\pi^{\mathrm{i}}$ contains $2 \underline{14} 3$, so $\pi=\left(\pi^{\mathrm{i}}\right)^{\mathrm{i}}$ contains $(2 \underline{14} 3)^{\mathrm{i}}=\begin{aligned} & 1 \overline{23} 4 \\ & 2143\end{aligned}$.

This generalizes to:

Proposition 3.2 Let $p$ be the pattern

$$
\cdots \underline{1 k} \cdots=(\cdots 1 k \cdots,\{j\}, \varnothing)
$$

in $S_{k}$, where $j=p^{\mathrm{i}}(1)$ is the index of 1 in $p$, so $j+1$ is the index of $k$ in $p$. A permutation $\pi$ in $S_{n}$ that avoids the pattern $p$ must also avoid the bivincular pattern

$$
\overline{12 \cdots \cdots} k=(\cdots 1 k \cdots, \varnothing,\{2,3, \ldots, k-2\})
$$

Proof: Assume a permutation $\pi$ contains the latter pattern in the proposition. If $1_{\pi}$ and $k_{\pi}$ are adjacent in $\pi$ we are done. Otherwise consider the element immediately to the right of $1_{\pi}$. If this element is larger than $(k-1)_{\pi}$ we replace $k_{\pi}$ by it and are done. Otherwise this element must me less than $(k-1)_{\pi}$ and therefore less than $2_{\pi}$, so we can replace $1_{\pi}$ by it, and repeat.

By applying the reverse to everything in sight in Proposition 3.2 we get:

Corollary 3.3 Let $p$ be the pattern

$$
\cdots \underline{k 1} \cdots=(\cdots k 1 \cdots,\{j\}, \varnothing)
$$

in $S_{k}$, where $j=p^{\mathrm{i}}(k)$ is the index of $k$ in $p$, so $j+1$ is the index of 1 in $p$. A permutation $\pi$ in $S_{n}$ that avoids the pattern p must also avoid the bivincular pattern

$$
\stackrel{1 \overline{2 \cdots \cdots} k}{\cdots \cdot k 1 \cdots}=(\cdots k 1 \cdots, \varnothing,\{2,3, \ldots, k-2\})
$$

By repeatedly applying the operations of inverse, reverse and complement we can generate six other corollaries. We will not need them here so we will not write them down.

Example 3.4 Let's look at some simple applications:

1. Consider the bivincular pattern $p_{1}=3 \underline{142}$. Proposition 3.2 shows a permutation $\pi$ that avoids $p_{1}$ must also avoid $1 \overline{23} 4$. In fact, the converse can be shown to be true, by taking inverses and applying the proposition. We will say more about the pattern $p_{1}$ in Example 3.5

2. Consider the bivincular pattern $p_{2}=3 \underline{15} 24$. The proposition shows that a permutation $\pi$ that avoids $p_{2}$ must also avoid $\begin{aligned} & 12345 \\ & 31524\end{aligned}$. We will say more about the pattern $p_{2}$ in subsection 3.2

Example 3.5 The Baxter permutations were originally defined and studied in relation to the "commuting function conjecture” of Dyer, see Baxter (1964), and were enumerated in Chung et al. (1978). Gire (1993) showed that these permutations can also be described as those avoiding the barred patterns $41 \overline{3} 52$ and 25̄135. It was then pointed out by Ouchterlony (2005) that this is equivalent to avoiding the vincular patterns $3 \underline{14} 2$ and $2 \underline{41} 3$. 
Similarly to what we did above we can show that the Baxter permutations can also be characterized as those avoiding the bivincular patterns $13 \overline{23} 4$ and $1 \overline{243} 4$, and this is essentially a translation of the description in Chung et al. (1978) into bivincular patterns.

Finally, here is an example that shows the converse of Proposition 3.2 is not true.

Example 3.6 The permutation $\pi=423165$ avoids the pattern ${ }_{23154}^{134}$ but contains the pattern $23 \underline{15} 4$, as the sub-word 23165.

\subsection{Gorenstein Schubert Varieties in terms of Bivincular Patterns}

Woo and Yong (2006) classify those permutations $\pi$ that correspond to Gorenstein Schubert varieties $X_{w}$. They do this using embeddings of patterns with Bruhat restrictions, which we have described above, and with a certain condition on the associated Grassmannian permutations of $w$, which we will describe presently:

First, a descent in a permutation $\pi$ is an integer $d$ such that $\pi(d)>\pi(d+1)$. A Grassmannian permutation is a permutation with a unique descent. Given any permutation $\pi$ we can associate a Grassmannian permutation to each of its descents, as follows: Given a particular descent $d$ of $\pi$ we construct the subword $\gamma_{d}(\pi)$ by concatenating the right-to-left minima of the segment strictly to the left of $d+1$ with the left-to-right maxima of the segment strictly to the right of $d$. More intuitively we start with the descent $\pi(d) \pi(d+1)$ and enlarge it to the left by adding increasing elements without creating another descent and similarly enlarge it to the right by adding decreasing elements without creating another descent. We then denote the flattening (or standardization) of $\gamma_{d}(\pi)$ by $\tilde{\gamma}_{d}(\pi)$, which is the unique permutation whose letters are in the same relative order as $\gamma_{d}(\pi)$.

Example 3.7 Consider the permutation $\pi=11|6| 12|94153728| 10$ where we have used the symbol $\mid$ to separate two digit numbers from other numbers. For the descent at $d=4$ we get $\gamma_{4}(\pi)=694578 \mid 10$ and $\tilde{\gamma}_{4}(\pi)=3612457$.

Now, given a Grassmannian permutation $\pi$ in $S_{n}$ with its unique descent at $d$ we construct its associated partition $\lambda(\pi)$ as the partition inside a bounding box $d \times(n-d)$, with $d$ rows and $n-d$ columns, whose lower border is the lattice path that starts at the lower left corner of the bounding box and whose $i$-th step, for $i \in \llbracket 1, n \rrbracket$, is vertical if $i$ is weakly to the left of the position $d$, and horizontal otherwise. We are interested in the inner corner distance of this partition, i.e., for every inner corner we add its distance from the left side and the distance from the top of the bounding box. If all these inner corner distances are the same then the inner corners all lie on the same anti-diagonal.

In Theorem 1 of Woo and Yong (2006) they show that a permutation $\pi \in S_{n}$ corresponds to a Gorenstein Schubert variety $X_{\pi}$ if and only if

1. for each descent $d$ of $\pi, \lambda\left(\tilde{\gamma}_{d}(\pi)\right)$ has all of its inner corners on the same anti-diagonal; and

2. the permutation $\pi$ avoids both 31524 and 24153 with Bruhat restrictions $\{(1 \leftrightarrow 5),(2 \leftrightarrow 3)\}$ and $\{(1 \leftrightarrow 5),(3 \leftrightarrow 4)\}$, respectively.

Let's take a closer look at condition 2 Proposition 3.8 below shows that avoiding 31524 with Bruhat restrictions $\{(1 \leftrightarrow 5),(2 \leftrightarrow 3)\}$ is equivalent to avoiding the bivincular pattern

$$
\begin{aligned}
& 12 \overline{34} 5 \\
& 31524
\end{aligned}=(31524,\{2\},\{3\}) .
$$


Similarly, avoiding 24153 with Bruhat restrictions $\{(1 \leftrightarrow 5),(3 \leftrightarrow 4)\}$ is equivalent to avoiding the bivincular pattern

$$
\begin{aligned}
& 1 \overline{23} 45 \\
& 24 \underline{15} 3
\end{aligned}=(24153,\{3\},\{2\}) \text {. }
$$

\section{Proposition $3.8 \quad$ 1. Let $p$ be the pattern}

$$
\cdots 1 k \cdots
$$

in $S_{k}$. Let $j=p^{\mathrm{i}}(1)$ be the index of 1 in $p$, so $j+1$ is the index of $k$ in $p$. A permutation $\pi$ in $S_{n}$ avoids $p$ with Bruhat restriction $(j \leftrightarrow j+1)$ if and only if $\pi$ avoids the vincular pattern

$$
\cdots \underline{1 k} \cdots=(\cdots 1 k \cdots,\{j\}, \varnothing) .
$$

2. Let $\ell \in \llbracket 1, k-1 \rrbracket$ and $p$ be the pattern

$$
\ell \cdots(\ell+1)
$$

in $S_{k}$. A permutation $\pi$ in $S_{n}$ avoids $p$ with Bruhat restriction $(1 \leftrightarrow k)$ if and only if $\pi$ avoids the bivincular pattern

$$
\begin{aligned}
& 1 \cdots \overline{\ell \ell+1} \cdots k \\
& \ell \cdots \ell \cdots+1
\end{aligned}=(\ell \cdots(\ell+1), \varnothing,\{\ell\})
$$

Proof: We consider each case separately.

1. Assume $\pi$ contains the vincular pattern mentioned. Then it clearly also contains an embedding satisfying the Bruhat restriction.

Conversely assume $\pi$ contains an embedding satisfying the Bruhat restriction. If $1_{\pi}$ and $k_{\pi}$ are adjacent then we are done. Otherwise look at the element immediately to the right of $1_{\pi}$. This element must be either larger than $k_{\pi}$, in which case we can replace $k_{\pi}$ by it and are done, or smaller, in which case we replace $1_{\pi}$ by it, and repeat.

2. Assume $\pi$ contains the bivincular pattern mentioned. Then it clearly also contains an embedding satisfying the Bruhat restriction.

Conversely assume $\pi$ contains an embedding satisfying the Bruhat restriction. If $(\ell+1)_{\pi}=\ell_{\pi}+1$ then we are done. Otherwise consider the element $\ell_{\pi}+1$. It must either be to the right of $(\ell+1)_{\pi}$ or to the left of $\ell_{\pi}$. In the first case we can replace $(\ell+1)_{\pi}$ by $\ell_{\pi}+1$ and be done. In the second case replace $\ell_{\pi}$ with $\ell_{\pi}+1$ and repeat.

As a consequence we get:

Corollary 3.9 A permutation $\pi$ in $S_{n}$ avoids

$$
\cdots 1 k \cdots,(j \leftrightarrow j+1)
$$

where $j$ is the index of 1 , if and only if the inverse $\pi^{-1}$ avoids

$$
j \cdots(j+1),(1 \leftrightarrow k) .
$$


Note that we could have proved the statement of the corollary with out going through bivincular patterns and then used that to prove part 2 of Proposition 3.8, as part 2 is the inverse statement of the statement in part 1

Translating condition 1 of Theorem 1 of Woo and Yong (2006) is a bit more work. The failure of this condition is easily seen to be equivalent to some partition $\lambda$ of an associated Grassmannian permutation $\tilde{\gamma}_{d}(\pi)$ having an outer corner that is either "too wide" or "too deep". Precisely, given a Grassmannian permutation $\pi$ and an outer corner of $\lambda(\pi)$, we say that it is too wide if the distance upward from it to the next inner corner is smaller than the distance to the left from it to the next inner corner. Conversely we say that an outer corner is too deep if the distance upward from it to the next inner corner is larger than the distance to the left from it to the next inner corner. We say that an outer corner is unbalanced if it is either too wide or too deep. We say that an outer corner is balanced if it is not unbalanced.

If a permutation has an associated Grassmannian permutation with an outer corner that is too wide we say that the permutation itself is too wide and similarly for too deep. If the permutation is either too wide or too deep we say that it is unbalanced, otherwise it is balanced. It is time to see some examples.

\section{Example 3.10}

1. Consider the permutation $\pi=14235$ with a unique descent at $d=2$. It corresponds to the partition (2) $\subseteq 2 \times 3$ and has just one outer corner. This outer corner is too wide.

2. Consider the permutation $\pi=13425$ with a unique descent at $d=3$. It corresponds to the partition $(1,1) \subseteq 3 \times 2$ and has just one outer corner. This outer corner is too deep.

3. Consider the permutation $\pi=134892567 \mid 10$ with a unique descent at $d=5$. It corresponds to the partition $(4,4,1,1) \subseteq 5 \times 5$ and has two outer corners. The first outer corner is too deep and the second is too wide.

4. Consider the permutation $\pi=13672458$ with a unique descent at $d=4$. It corresponds to the partition $(3,3,1) \subseteq 4 \times 4$ and has two outer corners that are both balanced.

These properties of Grassmannian permutations can be detected with bivincular patterns, as we now show.

Lemma 3.11 Let $\pi$ be a Grassmannian permutation.

1. The permutation $\pi$ is too wide if and only if it contains at least one of the bivincular patterns from the infinite family

$$
\mathcal{F}=\left(\begin{array}{l}
1 \overline{2345} \\
14235
\end{array}, 1 \overline{234567}, 1 \overline{23456789}, \ldots\right)
$$

The general member of this family is of the form

$$
\begin{aligned}
& 1 \overline{2 \cdots \cdots \cdot k} \\
& 1 \ell+1 \cdots 2 \cdot \ell k
\end{aligned}
$$

where $\ell=(k-3) / 2$. 
2. The permutation $\pi$ is too deep if and only if it contains at least one of the bivincular patterns from the infinite family

$$
\mathcal{G}=(\overline{1234} 5, \overline{123456} 7, \overline{12345678} 9, \ldots)
$$

The general member of this family is of the form

$$
\overline{12 \cdots \cdots \cdots k},
$$

where $\ell=(k-1) / 2$.

Proof: We only consider part 1, as part 2 is proved analogously. Assume that $\pi$ is a Grassmannian permutation that is too wide, so it has an outer corner that is too wide. Let $\ell$ be the distance from this outer corner to the next inner corner above. Then the distance from this outer corner to the next inner corner to the left is at least $\ell+1$. This allows us to construct an increasing sequence $t$ of length $\ell$ in $\pi$, starting at a distance at least two to the right of the descent. We can also choose $t$ so that every element in it is adjacent both in location and values. Similarly we can construct an increasing sequence $s$ of length $\ell$ in $\pi$, located strictly to the left of the descent. We can also choose $s$ so that every element in it is adjacent both in location and values. This produces the required member of the family $\mathcal{F}$.

Conversely, assume $\pi$ contains a particular member of the family $\mathcal{F}$. Then $\pi$ clearly has at least one outer corner that is too wide.

It should be noticed that these two infinite families are obtained from one another by reverse complement.

We have now shown that

Proposition 3.12 A permutation $\pi$ is balanced if and only if every associated Grassmannian permutation avoids every bivincular pattern in the two infinite families $\mathcal{F}$ and $\mathcal{G}$ in Lemma 3.11

This gives us:

Theorem 3.13 Let $\pi \in S_{n}$. The Schubert variety $X_{\pi}$ is Gorenstein if and only if

1. $\pi$ is balanced; and

2. the permutation $\pi$ avoids the bivincular patterns

$$
\begin{aligned}
& 12 \overline{34} 5 \text { and } 1 \overline{23} 45 \\
& 3 \underline{1524} \text { and } 24 \underline{15} 3 \text {. }
\end{aligned}
$$

I should note that with the descriptions of factorial and Gorenstein Schubert varieties given above it is easy to verify that smoothness implies factoriality implies Gorensteinness. 


\section{References}

E. Babson and E. Steingrímsson. Generalized permutation patterns and a classification of the Mahonian statistics. Sém. Lothar. Combin., 44:Art. B44b, 18 pp. (electronic), 2000. ISSN 1286-4889.

G. Baxter. On fixed points of the composite of commuting functions. Proc. Amer. Math. Soc., 15:851-855, 1964. ISSN 0002-9939.

M. Bousquet-Mélou and S. Butler. Forest-like permutations. Ann. Comb., 11(3-4):335-354, 2007. ISSN 0218-0006.

M. Bousquet-Mélou, S. Butler, A. Woo, and A. Yong. Personal communication between Bousquet-Mélou and Butler, and Woo and Yong, January 2005.

M. Bousquet-Mélou, A. Claesson, M. Dukes, and S. Kitaev. (2+2)-free posets, ascent sequences and pattern avoiding permutations. Journal of Combinatorial Theory Series A, 117:884-909, 2010.

F. R. K. Chung, R. L. Graham, V. E. Hoggatt, Jr., and M. Kleiman. The number of Baxter permutations. J. Combin. Theory Ser. A, 24(3):382-394, 1978. ISSN 0097-3165. doi: 10.1016/0097-3165(78)90068-7. URL http://dx.doi.org/10.1016/0097-3165(78) 90068-7.

S. Gire. Arbres, permutations à motifs exclus et cartes planaires: quelques problèmes algorithmique et combinatoire. $\mathrm{PhD}$ thesis, Université Bordeaux I, 1993.

V. Lakshmibai and B. Sandhya. Criterion for smoothness of Schubert varieties in $\mathrm{Sl}(n) /$ B. Proc. Indian Acad. Sci. Math. Sci., 100(1):45-52, 1990. ISSN 0253-4142.

E. Ouchterlony. On Young tableaux involutions and patterns in permutations. $\mathrm{PhD}$ thesis, Matematiska institutionen Linköpings universitet, Linköping, Sweden, 2005.

K. M. Ryan. On Schubert varieties in the flag manifold of $\operatorname{Sl}(n, \mathbf{C})$. Math. Ann., 276(2):205-224, 1987. ISSN 0025-5831. doi: 10.1007/BF01450738. URL http://dx.doi.org/10.1007/ BF 01450738 .

E. Steingrímsson. Generalized permutation patterns - a short survey. To appear in "Permutation Patterns, St Andrews 2007”, S.A. Linton, N. Ruskuc, V. Vatter (eds.), LMS Lecture Note Series, Cambridge University Press, 2007. URL/http: / / arxiv.org/abs/0801.2412v2.

J. West. Permutations with forbidden subsequences and stack-sortable permutations. PhD thesis, MIT, 1990.

J. S. Wolper. A combinatorial approach to the singularities of Schubert varieties. Adv. Math., 76(2):184193, 1989. ISSN 0001-8708. doi: 10.1016/0001-8708(89)90048-0. URL http://dx.doi.org/ $10.1016 / 0001-8708$ (89) 90048-0

A. Woo and A. Yong. When is a Schubert variety Gorenstein? Adv. Math., 207(1):205-220, 2006. ISSN 0001-8708.

A. Woo and A. Yong. Governing singularities of Schubert varieties. J. Algebra, 320(2):495-520, 2008. ISSN 0021-8693. 